\title{
Null fields in the outer Jovian magnetosphere: Ulysses observations
}

\author{
P. L. Haynes, A. Balogh, M. K. Dougherty, D. J. Southwood and A. Fazakerley \\ Space and Atmospheric Physics, Imperial College \\ E. J. Smith \\ Jet Propulsion Laboratory
}

\begin{abstract}
This paper reports on a magnetic field phenomenon, hereafter referred to as null fields, which were discovered during the inbound pass of the recent flyby of Jupiter by the Ulysses spacecraft. These null fields which were observed in the outer dayside magnetosphere are characterised by brief but sharp decreases of the field magnitude to values less than $1 \mathrm{nT}$. The nulls are distinguished from the current sheet signatures characteristic of the middle magnetosphere by the fact that the field does not reverse across the event. A field configuration is suggested that accounts for the observed features of the events.
\end{abstract}

\section{Introduction}

The magnetic field in the dayside outer magnetosphere of Jupiter is subject to large scale dynamic variations. The inbound pass of Ulysses took place at a similar local time to the previous flybys by the Pioneer and Voyager spacecraft, namely the mid-morning sector of the Jovian magnetosphere at relatively low latitude. During the recent flyby in early February 1992, Ulysses first detected the magnetopause at a distance of $110 R_{J}\left(R_{J}=\right.$ Jovian radius), the furthest encounter yet reported by a spacecraft. During the inbound pass, Ulysses made multiple crossings of the magnetopause, the last being on 4 February 1992 at about 02:50 UT at a distance of $88 R_{J}$ (Balogh et al., 1992; Bame et al., 1992). In this paper we examine a class of dramatic magnetic field events which occur in the outer magnetosphere in which the field magnitude drops to a small fraction of the ambient value. We call these events nulls.

Using the Pioneer magnetometer data, Smith et al, (1976) describe the dayside magnetosphere of Jupiter as divided into three major regions based on the field morphology: Inner, middle and outer magnetosphere. In the inner magnetosphere the field is dominated by the planetary dipole. The field in the middle magnetosphere is dominated by the current sheet (or magnetodisk), an intense ring of current flowing around the planet roughly in the magnetic equatorial plane. The field is mainly radially directed with a substantial azimuthal component. In the outer magnetosphere, the near equatorial

Copyright 1994 by the American Geophysical Union.

Paper number 93GL01986

0094-8534/94/93GL-01986\$03.00 field is highly disturbed and predominantly in the direction parallel to the planetary dipole. The null field phenomena that we report here are detected in this outer region. The events are characterised by brief but sharp decreases of the field magnitude to values less than $1 \mathrm{nT}$ in a background field of approximately $7 \mathrm{nT}$, and are distinct from current sheet crossings in the middle magnetosphere which also give rise to regions of low field magnitude in the centre. The nulls reported here occur in a region where the field at Ulysses is primarily southward (parallel to the planetary dipole). Current sheet encounters occur where the dominant field component is in the radial direction. However the clearest distinction between the low field region in the centre of a current sheet is that the field reverses sense across the sheet whereas in the nulls reported here the field returns outside the null region to the same direction as before the encounter; there is no field reversal. Our interpretation is that the null region is populated by detached "blobs" of plasma whose pressure is sufficient to exclude the outer magnetospheric field. The plasma is likely to have been detached from the current sheet but further investigation is required to establish the process. A reference to sporadic occurrences of plasma density enhancements associated with field depressions in the outer magnetosphere suggests that the null field events were also detected by the Voyager spacecraft (Belcher, 1983).

\section{Overview of the Ulysses Inbound Pass}

In Figure 1 we show 36 hours worth of magnetometer data from the Ulysses inbound pass (Balogh et al., 1992). Three field components and the field magnitude are displayed in a right-handed spherical polar coordinate system $(r, \theta, \phi)$ whose $z$-axis is northward aligned with the (centred) planetary dipole. The radial component corresponds to the component in the direction away from the centre of the planet. The $B_{\theta}$ component is in the direction of increasing magnetic latitude (as measured from the planetary dipole) and the azimuthal component $\left(B_{\phi}\right)$ is in the direction of planetary rotation. The lowest panel shows the magnitude of the field. Throughout the region the field is primarily in the $B_{\theta}$ direction; this is characteristic of the outer magnetosphere (as defined by Smith et al., 1976).

The last encounter with magnetosheath plasma is marked in the magnetometer data by a depression in the field strength between 01:00 and 03:00 UT on 4 February 1992 
JOVICENTRIC DISTANCE

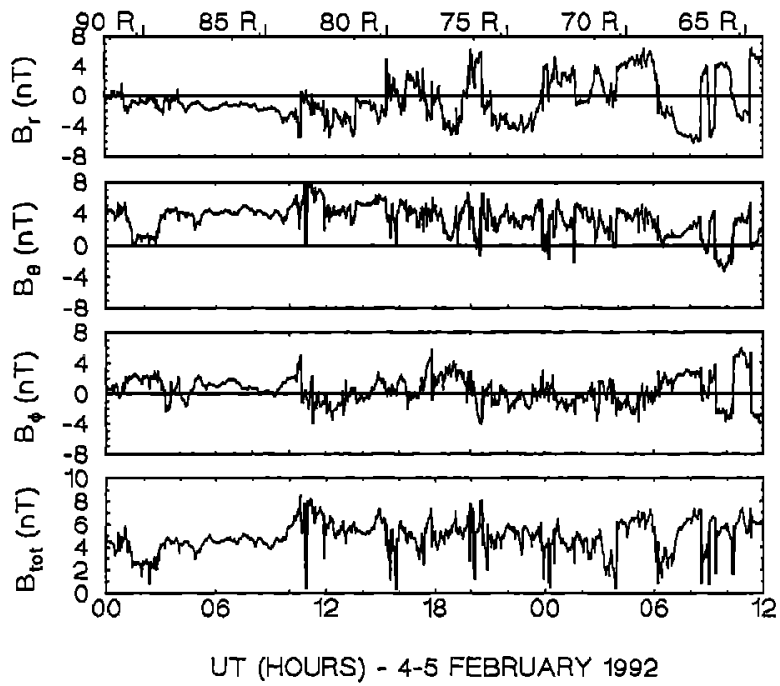

Fig. 1. Thirty six hours worth of magnetometer data (48 second averages) from the Ulysses inbound pass are shown versus time, where the 3 field components (in the planetary magnetic coordinate system defined in the text) and the field magnitude are displayed. Null fields events are seen on 4 February 1992 at 10:54, 15:31, 15:52, 23:57 UT, and on 5 February 1992 at $00: 14,00: 16$, and 03:49 UT. Decreases in field magnitude occuring after 06:00 UT on 5 Feburary are due to encounters with the distorted outer edge of the current sheet.

(Bame et al., 1992). In the early part of the plot the dominant field component is in the $\theta$ direction. By the end of the period the dominant field component is the radial component. Thus the plot covers the transition from the outer magnetosphere to the middle magnetosphere.

In the lower panel which displays the field magnitude, there are sporadic brief decreases of the field to less than $1 \mathrm{nT}$. These are the events which we call nulls. When viewed in high resolution, events are seen on 4 February 1992 at 10:54, 15:31, 15:52, 23:57 UT, and on 5 February 1992 at 00:14, 00:16, and 03:49 UT. In all cases, the field magnitude decreases below $1 \mathrm{nT}$; in some cases the field becomes as small as 0.2 or $0.1 \mathrm{nT}$. The drop in field magnitude on 4 February at 20:13 UT does not show the characteristics associated with all the other null events; the magnitude only decreases to $\sim 1.2 \mathrm{nT}$ and the directional behaviour is ambiguous (see Section 3.). Decreases in field magnitude occurring after 06:00 UT on 5 February 1992 are due to encounters with the distorted outer edge of the current sheet.

\section{Null Field Signature}

Figure 2 shows an example of the field in the null region shown at higher resolution. Forty minutes of data are shown taken from 4 February 1992, when the spacecraft was some $82 R_{J}$ from the planet. The field vectors are shown at a time resolution of 1 second and are plotted in
JOVICENTRIC DISTANCE

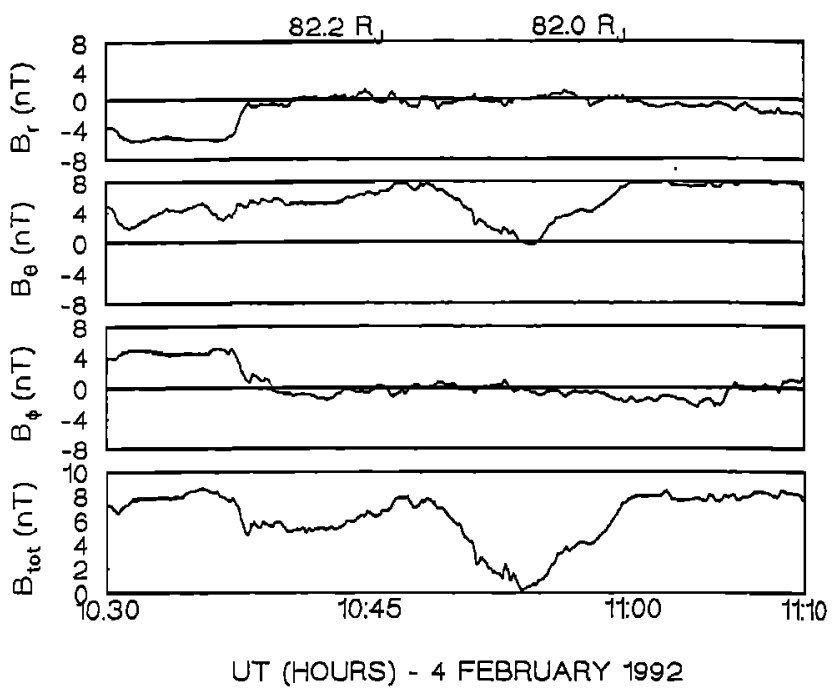

Fig. 2. Forty minutes worth of high resolution (1 second) magnetic field data from 4 February 1992 showing the occurrence of a null field.

the same magnetic coordinate system as Figure 1 . The layout of the panels is the same as in Figure 1.

The dominant feature of the plot is the decrease of the $\theta$ component from a value of order $7 \mathrm{nT}$ outside the null to less than $1 \mathrm{nT}$ at the field magnitude minimum at the centre of the event at 10:54 UT. Comparison of the $B_{\theta}$ component plot and the field magnitude shows that throughout the event, except briefly near the centre, the field is predominantly in the $\theta$ direction.

The radial and azimuthal components are small in magnitude $(\sim 2 \mathrm{nT})$ throughout and are subject to fluctuations on periods of order one minute or so. Both the components transverse to the main field (in the $\theta$ direction) reverse sign through the centre of the event at 10:54 UT. In contrast, the $B_{\theta}$ component is close to symmetrical about the centre of the null. It should be noted that the $B_{\theta}$ component is non zero at the centre of the null. In fact the component briefly reverses; a northward $B_{\theta}$ of $0.20 \pm 0.05 \mathrm{nT}$ is detected shortly after the minimum in the field. We suspect that this weak field reversal near the centre is symptomatic of the internal structure of the null event.

Following the event, the $B_{\theta}$ component increases rapidly and resumes its original value outside the null. The spacecraft is near the magnetic equatorial plane for the entire inbound pass and thus when the azimuthal component becomes negative it implies that the field is being bent away from the rotation direction, when it is positive it is being bent towards the rotation direction. Similarly, the bending of the radial component is such that the field is tilted towards the planet just before the middle of the event and away after the central passage. The azimuthal and radial field components remain small in magnitude but fluctuate. There are also fluctuations in the $B_{\theta}$ component but with smaller magnitude than in the transverse directions. The disturbances thus appear 
to be mainly transverse to the field and thus are likely to be MHD waves propagating in the intermediate (Alfvén) mode.

As the spacecraft continues further in towards the planet regions of low field magnitude were detected at times when the equatorial current sheet is periodically encountered. In contrast with the nulls reported here, the $B_{\theta}$ component is either negligible before, after and during these later current sheet crossings or clearly reverses sign through the event. The former signature corresponds to "classical" current sheet encounters across which the primarily radial field of the middle magnetosphere reverses. The latter type of encounter (in which the ambient field contains a significant $B_{\theta}$ component) correspond to encounters with a severely displaced, rippled or warped sheet and will form the subject of a future study. The sharp decreases in field magnitude occurring between 06:00 and 12:00 UT on 5 February 1992 (see Figure 1) may be regarded as crossings of a distarted current sheet.

\section{Interpretation}

In Figure 3, we sketch a field configuration that might represent the field in the vicinity of the nulls reported here. We propose that the events are caused by the passage of the spacecraft through a tube of enhanced plasma aligned roughly along the direction of the ambient field, i.e. in the $\theta$ direction. Manifestly in the centre of the events there is strong deficiency in the field pressure which we assume must be made up by plasma pressure. Thus we predict that the net number density, temperature or both must increase as the field pressure decreases.

Such a "blob" or bubble of plasma detached from the planet could form by material breaking off at the outer edge of the current sheet in the middle magnetosphere. All the events reported here have been detected in the outer magnetosphere beyond the radial distance where the current sheet appears to be present. We thus propose that the field configuration in Figure 3 can be understood by envisaging a "blob" or bubble of plasma detached from the sheet pushing apart the mainly southward field of the outer magnetosphere as it moves radially. The field lines are pushed apart by the plasma pressure of the "blob". The pressure required to exclude the outer magnetospheric field $(\sim 7 \mathrm{nT})$ is similar to that required in the centre of the middle magnetospheric current sheet as the field strength in the vicinity of the first current sheet encounters made by Ulysses is comparable to 7 $\mathrm{nT}$. It follows that it may not be necessary to invoke a major degree of heating or acceleration in the process of "blob" formation.

Our sketch shows the plasma enhancement with a "cigar-like" shape with its axis along the background field direction. The orientation is consistent not only with the configuration of the external field in the outer magnetosphere but also is appropriate to account for the transverse field displacements in the $B_{r}$ and $B_{\phi}$ components which reverse in sign through the centre of the event.

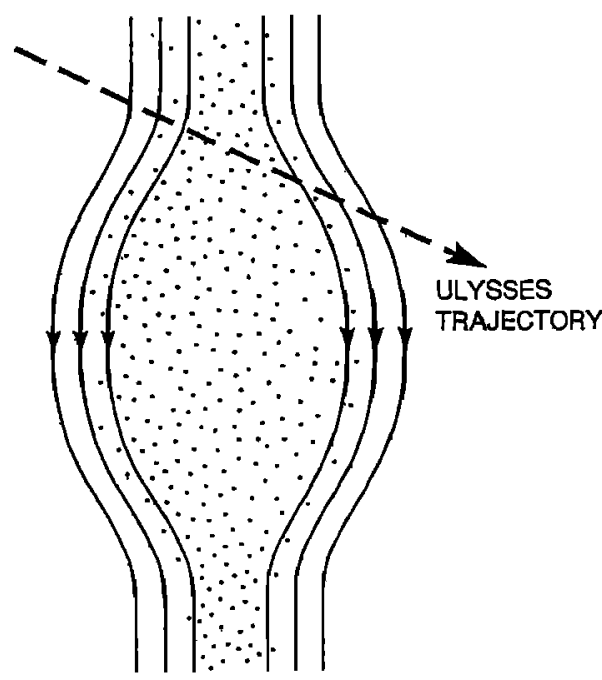

Fig. 3. The possible field and plasma configuration encountered by Ulysses in the vicinity of a null field. The solid lines represent the magnetic field and the dotted region the plasma.

\section{Conclusions}

We have reported a new discovery made with the Ulysses spacecraft magnetometer. Field nulls have been detected with the Ulysses spacecraft in its passage through the extended outer dayside magnetosphere during the Jupiter flyby in February 1992. A passing reference to the occurrence of similar phenomena encountered during the Voyager flybys of Jupiter is made by Belcher (1983). Belcher notes the sporadic appearances of cold, dense concentrations of electrons and ions which correlate with decreases in magnetic field strength. We plan to work with other Ulysses instrument teams to examine the relationship between field and plasma and possibly re-examine the data from previous flybys.

Belcher's remark concerns "blobs" of plasma on the dayside in a similar region to where our null fields originate. In a nightside context it is interesting to note Ansher et al.(1992) which reports on density structures detected on the outbound pass of the Voyager 1 spacecraft. Yang et al.(1992) suggest that material is driven centrifugally outward from the Io torus in the form of large "fingers" of plasma. Whether such structures could propagate with little change all the way to the outer magnetosphere deserves further investigation.

The outer magnetosphere was defined by Smith et al. (1976) as where the field is primarily southward (in the $\theta$ direction) and the null events are characterised by the $B_{\theta}$ component dropping to a small value or even reversing slightly but then returning to the same sign and value after the event as before. Our proposed explanation of the events is that the spacecraft has flown through regions of enhanced plasma pressure elongated along the ambient field direction. We suggest that the material in the core of the null originates in the current sheet closer to the planet. The breaking off of material from the equato- 
rially confined centrifugally accelerated material which would tend to move away from the planet seems possible, say, after a sudden decrease in external pressure. Further theoretical investigation is planned.

Acknowledgements The Ulysses-related work at Imperial College, London is supported by the U.K. Science and Engineering Research Council who also fund the Research Studentship of PLH. Work at the Jet Propulsion Laboratory of the California Institute of Technology has been carried out under contract to NASA.

\section{References}

Ansher, J. A. , W. S. Kurth, D. A. Gurnett, In Memoriam C. K. Goertz, "High Resolution Measurements of Density Structures in the Jovian Plasma Sheet", Geophys. Res. Lett., 19, 23, 2282-2284, 1992.

Balogh, A. , M. K. Dougherty, R. J. Forsyth, D. J. Southwood, E. J. Smith, B. T. Tsurutani, N. Murphy,

M. E. Burton, "Magnetic Field Observations during the Ulysses flyby of Jupiter", Science 257, 1515-1518, 1992.

Bame, S. J. , B. L. Barraclough, W. C. Feldman, G. R. Gisler, J. T. Gosling, D. J. McComas, J. L. Phillips,
M. F. Thomsen, B. E. Goldstein, M. Neugebauer, "Jupiter's Magnetosphere: Plasma Description from the Ulysses Flyby", Science 257, 1539-1543, 1992.

Belcher, J. W. "The low-energy plasma in the Jovian magnetosphere", in Physics of the Jovian Magnetosphere, ed. A. J. Dessler, Cambridge Planetary Science Series, 68-105, 1983.

Smith, E. J. , L. Davis, and D. E. Jones, "Jupiter's magnetic field and magnetosphere", in Jupiter, ed. T. Gehrels, University of Arizona Press, 788-828, 1976.

Yang, Y. S. , R. A. Wolf, R. W. Spiro, A. J. Dessler, "Numerical Simulation of Plasma Transport driven by the Io Torus", Geophys. Res. Lett., 19, 10, 957-960, 1992.

A. Balogh, M.K. Dougherty, A. Fazakerley, P.L. Haynes, D.J. Southwood, Space and Atmospheric Physics, Imperial College, London SW7 2BZ, UK.

E.J. Smith, Jet Propulsion Laboratory, California, CA 91109, USA.

(Received 29 October 1992;

revised 22 March 1993;

accepted 18 June 1993.) 